\title{
El profesorado de Educación Infantil y Primaria: formación tecnológica y competencia digital
}

\author{
Pre-school and primary school teachers: \\ technological training and digital competence
}

RECIBIDO 30/3/2021 ACEPTADO 16/6/2021 PUBLICADO 1/12/2021

\section{Vicente Gabarda Méndez}

Departamento de Didáctica y Organización Escolar, Universidad de Valencia, España

vicente.gabarda@uv.es

\section{(iD) Enrique García Tort}

Departamento de Didáctica y Organización Escolar, Universidad de Valencia, España

gartort@alumni.uv.es

(iD) María de Lourdes Ferrando Rodríguez

Área de educación, Universidad Internacional de Valencia, España

mariadelourdes.ferrando@campusviu.es

iD Andrés Chiappe Laverde

Centro de Tecnologías para la Academia, Universidad de La Sabana, Colombia

andres.chiappe@unisabana.edu.co

\section{RESUMEN}

En una sociedad cada vez más digital, la escuela está llamada a cumplir una responsabilidad fundamental en el desarrollo de la destreza tecnológica de los estudiantes de cualquier etapa educativa. El papel del docente es, por tanto, un factor clave para el desarrollo de la competencia digital, especialmente, en las primeras edades. En este sentido, este artículo pretende analizar, mediante una revisión normativa y bibliográfica, cómo se ha ido integrando la tecnología en nuestro sistema educativo y qué importancia ha tenido la formación tecnológica del profesorado en los últimos años. Asimismo, se revisan diferentes propuestas de marcos de competencia digital docente, a fin de poder contraponer la formación inicial con las destrezas que se le requiere al profesorado por parte de diferentes organismos nacionales e internacionales. Para finalizar, se reflexiona acerca del nivel de competencia digital docente del profesorado en formación y en ejercicio en la actualidad, identificando puntos fuertes y débiles en relación a sus responsabilidades curriculares y a los marcos anteriormente examinados. Este análisis permite poner de relieve que, aunque se ha recorrido un camino importante en esta línea, aún queda mucho por hacer, especialmente en los planes de formación inicial donde la promoción de las destrezas digitales y tecnológicas de los futuros docentes es aún una asignatura pendiente.

PALABRAS CLAVE competencia digital, formación del profesorado, educación infantil, educación primaria. 


\section{ABSTRACT}

In an increasingly digital society, schools have a fundamental responsibility in developing the technological skills of students at all stages of education. The role of the teacher is, therefore, a key factor in the development of digital competence, especially at an early age. In this sense, this article aims to analyze, through a normative and bibliographical review, how technology has been integrated into our educational system and the importance of technological training for teachers in recent years. It also reviews different proposals for teacher digital competence frameworks in order to compare initial training with the skills required of teachers by different national and international organizations. Finally, we reflect on the level of digital competence of today's trainee and practicing teachers, identifying strengths and weaknesses in relation to their curricular responsibilities and the frameworks discussed above. This analysis highlights the fact that, although significant progress has been made in this area, there is still much to be done, especially in initial training plans where the promotion of digital and technological skills among future teachers is still an unresolved issue.

KEYWORDS digital competence, teacher training, childhood education, primary education.

\section{INTRODUCCIÓN}

La educación se encuentra, en la actualidad, en un momento de revolución. Esta revolución, aunque viene gestándose desde hace varios años, ha tenido un punto de inflexión de enorme trascendencia: la pandemia derivada de la COVID-19. Esta situación sobrevenida ha generado una aceleración de los procesos de reflexión acerca de cómo se diseñan, implementan y evalúan los procesos formativos y ha puesto de manifiesto algunas de las grandes carencias de cualquier sistema educativo (Cuevas et al., 2020).

Al margen de cuestiones vinculadas al equipamiento, la capacidad del profesorado para adaptarse a una nueva realidad que exigía llevar a cabo procesos de enseñanza híbrida o en línea ha sido una de las cuestiones que más debate y preocupación ha generado en la comunidad educativa y la literatura científica.

Para entender mejor esta situación, el artículo que se presenta a continuación pretende fundamentar, desde un punto de vista histórico y normativo cómo que ha venido contemplando en los últimos años la formación inicial del profesorado de Educación Infantil y Primaria. Este análisis permitirá ver, entre otras cuestiones, cómo se han integrado los contenidos tecnológicos en la escolaridad como punto de partida de la necesidad de que los docentes adquiriesen competencias de carácter tecnológico. Además, se pondrá de relieve la importancia que se le ha concedido al desarrollo de la competencia digital docente en los planes de formación inicial, a fin de tener una visión panorámica de las destrezas promovidas por la administración educativa. Además, se presentarán aproximaciones a marcos de competencia digital, una cuestión muy relevante para poder valorar con mayor criterio la idoneidad de la formación promovida. Todas estas prescripciones serán contrapuestas, en la parte final del manuscrito, con los niveles de competencia digital de los docentes en formación y en ejercicio según la literatura científica de los últimos años. 


\section{LA FORMACIÓN INICIAL DOCENTE EN MATERIA TECNOLÓGICA: PERSPECTIVA HISTÓRICA Y NORMATIVA}

Tras la formación del Espacio Europeo de Educación Superior (EEES) en 1999, se consolidó entre los países miembros la preocupación por mejorar e integrar las tecnologías en el aula. Así pues, el marco supranacional generó la oportunidad para que se pusiera en marcha en España una cadena evolutiva de reformas de la legislación educativa y los planes de formación inicial del profesorado con tal de ajustarse a las demandas de una nueva sociedad y al creciente auge las Tecnologías de la Información y la Comunicación (TIC) (Herrada, \& Herrada, 2011).

Dichos cambios y adaptaciones adquirieron un especial sentido con el desarrollo de las Competencias Clave para el Aprendizaje Permanente, planteadas por la Comisión Europea (2006) y revisadas por el Consejo de la Unión Europea en 2018. Dentro de estas Competencias Clave se encuentra recogida la Competencia Digital, que vertebró la formación inicial docente y las propuestas legislativas en España relacionadas con la tecnología y la educación (Aristizabal, \& Cruz, 2018).

Estas directrices trataban de formalizar, mediante el reconocimiento explícito de la competencia digital, una necesidad creciente derivada de la progresiva incorporación de las TIC en los sistemas educativos en las últimas décadas. En el marco legislativo español, y de manera paralela a las cuestiones de equipamiento físico, la presencia curricular de la tecnología es visible desde la Ley General de Educación (LGE, 1970), donde se introdujo la asignatura optativa de informática. Su abordaje era, sin embargo, escaso, dado que se impartía fuera de la educación obligatoria, con el consecuente ataque a la comprensividad de la educación tecnológica y la competencia digital.

Tras la llegada de la Ley Orgánica de Ordenación General del Sistema Educativo (LOGSE, 1990) comenzaron las primeras alusiones al uso de herramientas audiovisuales y ordenadores. La LOGSE también trajo consigo la aparición del área de Tecnología en la Educación Secundaria Obligatoria, quien fue la encargada de abordar los contenidos relacionados con las TIC.

Impulsada por las Competencias Clave para el Aprendizaje Permanente citadas anteriormente, la Ley Orgánica de Educación (LOE, 2006) contempló las competencias en las diferentes etapas, siendo la primera ley educativa que recoge el término "competencia digital" en sus principios pedagógicos. A través de la LOE se promovió la mejora del acceso a las TIC, la introducción de Entornos Virtuales de Aprendizaje como recurso educativo y la promoción y uso de las TIC como medio didáctico. Respecto al cuerpo docente, la LOE señalaba la necesidad de mejorar la capacitación docente y de desarrollar las aptitudes, conocimientos y competencias necesarias para la sociedad del conocimiento.

De forma complementaria a la LOE (2006), se promovieron normativas curriculares para cada etapa que permitieron materializar de un modo más concreto la integración de las TIC en los procesos formativos. En el caso de la Educación Infantil, el Real Decreto 1630/2006 reconocía la necesidad de promover entre los estudiantes una primera aproximación a las TIC, mientras que el Real Decreto 1513/2006 (modificado por el Real Decreto 126/2014) marcó la Educación Primaria como la encargada de iniciar en la utilización y el aprendizaje de las TIC a través de un espíritu crítico junto a la integración transversal de las TIC en las diferentes asignaturas.

La Ley Orgánica para la Mejora de la Calidad Educativa (LOMCE, 2013) reconoce en su preámbulo que el sistema educativo se enfrenta a nuevos retos generacionales donde la globalización y el impacto de las 
nuevas tecnologías han provocado una nueva manera de aprender y comunicarse. Desde esta premisa, se conciben las TIC como una serie de herramientas que permiten personalizar la educación en ritmo y necesidades, gestionar los centros y mejorar los procesos formativos. De este modo, las TIC se convierten en una herramienta clave indispensable para la formación del profesorado y para el desarrollo de las competencias digitales del alumnado. No obstante, la LOMCE ha sido recientemente sustituida por la Ley Orgánica por la que se Modifica la Ley Orgánica de Educación (LOMLOE, 2020).

Al igual que la LOE recogió en su desarrollo la influencia de las Competencias Clave para el Aprendizaje Permanente elaboradas en 2006 por la Comisión Europea, la LOMLOE acoge en su propuesta pedagógica la revisión de las Competencias Clave del 2018. Por ende, la se reconoce el uso generalizado de las TIC y de la importancia de su integración en la educación. Desde esta perspectiva, y acorde al papel clave en el desarrollo humano de las Competencias Digitales, en su preámbulo se incita un cambio de enfoque que reconozca el impacto social y personal de la tecnología (LOMLOE, 2020).

Esta normativa, que entrará en vigor en el curso 2021/2022 y que, por tanto, no ha promovido hasta el momento normativas que establezcan nuevas concreciones curriculares, respeta en su planteamiento general la consideración de la etapa de Educación Infantil como una primera aproximación a las tecnologías. Sin embargo, en Educación Primaria y etapas posteriores, la LOMLOE añade al marco normativo la preocupación por los riesgos derivados del uso de las TIC. Es decir, contemplar el desarrollo de la Competencia Digital en docentes y alumnado no sólo como el acceso y el manejo de tecnologías, sino también como la prevención de mala utilización de las TIC y la formación en seguridad:

“Las Administraciones educativas deberán establecer las condiciones que hagan posible la eliminación en el ámbito escolar de las situaciones de riesgo derivadas de la inadecuada utilización de las TIC, con especial atención a las situaciones de violencia en la red" (LOMLOE, 2020).

De esta forma, la desigualdad en el uso de las TIC se hace visible, tratando de romper la brecha de género en el uso de las tecnologías e incrementando la presencia femenina en las áreas de Ciencia y Tecnología. En suma, las TIC son entendidas como una herramienta para combatir la discriminación y afrontar los nuevos retos educativos.

Parecería obvio, con este desarrollo progresivo de las TIC en las directrices supranacionales y las normativas estatales de carácter general y curricular, que el desarrollo de la formación tecnológica del profesorado de estas etapas forma parte inherente de las reformas.

En efecto, el reconocimiento de la competencia digital como competencia clave para el aprendizaje permanente de cualquier ciudadano en 2006 supuso un impulso de la promoción de las destrezas tecnológicas del profesorado. De este modo, la competencia digital y las TIC fueron incorporadas a la normativa que regulaba la formación inicial del profesorado de Educación Infantil y de Educación Primaria.

Por un lado, la Orden ECI/3854/2007, de 27 de diciembre, por la que se establecen los requisitos para la verificación de los títulos universitarios oficiales que habiliten para el ejercicio de la profesión de Maestro en Educación Infantil planteó como objetivo "conocer las implicaciones educativas de las tecnologías de la información y la comunicación y, en particular, de la televisión en la primera infancia”. Además, estableció dentro como competencias en la formación inicial el análisis de campo observacional a través de las tecnologías de la información, documentación y audiovisuales, así como el fomento de experiencias de iniciación 
a las TIC. Aunque los planteamientos de esta normativa podrían responder a una realidad contextualizada en 2007, cabe destacar que sigue siendo la regulación aplicable a la formación inicial en Educación Infantil, siendo claro el desajuste tecnológico con el contexto actual donde constituyen elementos básicos los EVA, las tabletas, los ordenadores, los smartphones o demás dispositivos actuales.

Respecto a la regulación de la formación inicial en Educación Primaria, la Orden ECl/3857/2007, de 27 de diciembre, por la que se establecen los requisitos para la verificación de los títulos universitarios oficiales que habiliten para el ejercicio de la profesión de Maestro en Educación Primaria planteó entre sus objetivos conocer y aplicar en las aulas las TIC, así como ser capaz de discernir aquella información que contribuya positivamente al aprendizaje, la formación cívica y la riqueza cultural. Sin embargo, aunque la regulación de la formación inicial en Educación Primaria reconoció la mutua influencia entre ciencia, sociedad y desarrollo tecnológico, el documento no hace mención explícita en las TIC, las herramientas tecnológicas o la Competencia Digital entre las competencias que el profesorado debe adquirir tras la formación inicial.

Esta carencia explícita de contenidos y destrezas tecnológicas en la normativa reguladora de la formación inicial de los docentes de las etapas que nos ocupan ha generado una integración desigual de la Competencia Digital en la formación del profesorado entre diferentes planes de estudio (Gabarda et al., 2020), llegando a suponer, en la mayor parte de los casos, una disminución de los contenidos tecnológicos en los planes de formación inicial tras la entrada en vigor del EEES (Herrada, \& Herrada, 2011; Peirats et al., 2018).

\section{MARCOS DE REFERENCIA EN COMPETENCIA DIGITAL}

Tal y como planteábamos en el apartado anterior, la progresiva integración de las TIC en las diferentes esferas de nuestras vidas puso de relieve la necesidad de establecer marcos supranacionales desde los que regular su utilización. En este sentido, y una vez reconocida la competencia digital como una de las competencias clave que deben ser desarrolladas al culminar la enseñanza obligatoria para la incorporación del alumnado a la vida adulta de forma satisfactoria, comenzaba el reto de poder implementar líneas específicas para su fomento en diferentes escenarios y contextos. Sin embargo, intentar definir las competencias digitales desde una perspectiva meramente instrumental es limitar no solo su definición, sino sus implicaciones en la vida cotidiana de ciudadanos y ciudadanas que utilizan la tecnología en su día a día.

Desarrollar la competencia digital en el sistema educativo, supone integrar el uso de las TIC en las aulas y que el personal docente posea formación en esta competencia cada vez más necesaria y demandada en un mundo, cada vez más digital.

La necesidad de centrar la mirada en marcos de referencia y estándares que permitan avanzar en el diseño de propuestas de formación y acreditación de las competencias digitales docentes, invitan a analizar algunas iniciativas y documentos base promovidos por organismos internacionales y adoptados por algunos países, en materia de competencia digital.

La Organización de Naciones Unidas para la Educación, la Ciencia y la Cultura (UNESCO) impulsó en 2008 un marco general para el establecimiento de estándares en competencias TIC para el docente, combinando tres factores que se consideraron básicos para la transformación en la educación: la alfabetización tecnológica, la profundización de conocimientos, y la creación de conocimiento. Estos tres factores, relacionados con seis componentes del sistema educativo como: la política, el Curriculum y plan de estudios, la pedagogía, las TIC, la organización y gestión, y la formación y desarrollo de los docentes, (Esteve-Mon, 
et al., 2016) fueron importantes elementos que ayudaron a avanzar en reflexiones y propuestas derivadas. La UNESCO actualizó este marco en 2019, identificando seis áreas (comprensión del papel de las TIC en las políticas educativas, currículo y evaluación, pedagogía, aplicación de competencias digitales, organización y administración y aprendizaje profesional de los docentes) a desarrollar en tres niveles (adquisición de conocimientos, profundización de conocimientos y creación de conocimientos), ofreciendo una perspectiva más actualizada y ajustada a las necesidades presentes.

Al margen de la UNESCO, proliferaron hace alrededor de una década propuestas por parte de otros organismos como la Organización para la Cooperación y el Desarrollo Económico (OCDE), la European Computer Driving Licence Foundation (Fundación ECDL) o a la International Society for Technology in Education (ISTE), orientadas a desarrollar una serie de estándares o lineamientos en materia digital. Sin embargo, tal y como apuntan Ramírez et al. (2015) las directrices de todos ellos plantean enfoques diversos. Mientras que la OCDE y la UNESCO aportan una visión económica y educativa, la ECDL aporta un enfoque de estandarización y certificación de competencias en TIC con perspectiva global y la ISTE, una propuesta de corte más regional por centrarse en el contexto estadounidense.

Centrando nuestra atención en la propuesta de la International Society for Technology in Education, ésta trató de ofrecer un marco, para que cada administración pudiera articular planes propios de desarrollo y así planificar una adecuada formación del profesorado en esta materia. Su propuesta de Estándares de Tecnologías de la Información y la Comunicación para docentes (NETS-T) de 2008, constituyó un intento de describir la competencia digital docente a través de la inclusión de matrices de valoración para el profesorado de la era digital, ofreciendo ejemplos de criterios de desempeño para niveles incrementales de logro: el nivel principiante, para describir los niveles de desempeño esperados en estudiantes que cursan programas de formación de docentes, o en maestros en práctica que se inician en el uso de las TIC, para mejorar la enseñanza y el aprendizaje; el nivel medio, para describir comportamientos esperados de profesorado que está adquiriendo más experticia y flexibilidad en la utilización de las TIC en entornos educativos; el nivel experto, para describir comportamientos que demuestran que el personal docente está usando las TIC de forma eficiente y efectiva. Por último, el nivel transformador, como el nivel que describe comportamientos que conllevan explorar, adaptar y aplicar las TIC de forma que cambian fundamentalmente la enseñanza y el aprendizaje. Esta propuesta permitía, por tanto, sentar las bases para que el profesorado lograra evidenciar competencias que facilitaran el aprendizaje y la creatividad de su alumnado, diseñar y desarrollar experiencias de aprendizaje y evaluaciones propias de la era digital, modelar el trabajo y aprendizaje característicos de la era digital, promover y ejemplificar su ciudadanía digital y responsabilidad, su compromiso con el crecimiento profesional y con el liderazgo.

Por su parte, el gran avance en relación a la identificación de competencias digitales docentes en el contexto comunitario, vino de la mano del Marco Europeo de Competencias Digitales para la Ciudadanía (DigComp), una herramienta desarrollada por la Comisión Europea (Ferrari, 2013). Este marco, no solo se convirtió en 2013 en un referente en cuanto al desarrollo y la planificación de iniciativas en materia de competencia digital, tanto a escala europea, como de los Estados miembros, sino que se convirtió en la antesala al marco DigCompEdu, un marco específico para la competencia digital docente de todos los niveles de la educación, desde la primera infancia hasta la educación superior y de adultos, incluida la formación general y profesional, la educación para las necesidades especiales y los contextos de aprendizaje no formal (Redecker, \& Punie, 2017). Su objetivo, fue proporcionar un marco de referencia general para los desarrolladores 
de modelos de competencia digital, como pueden ser, los gobiernos y organismos nacionales y regionales, las organizaciones educativas, los proveedores de formación profesional, y los propios educadores. Se establecieron niveles de competencia ya utilizados por el Marco Común Europeo de Referencia para las Lenguas, contemplándose niveles desde A1 (Novato) hasta C2 (Pionero).

El DigCompEdu, considera seis áreas de competencias diferentes con un total de 22 competencias. Mientras que el área 1 se centra en el entorno profesional, él área 2 se vincula con las fuentes, la creación y la distribución de recursos digitales. Por su parte, el área 3 se relaciona con la administración y el uso de herramientas digitales en la enseñanza y el aprendizaje y el área 4 aborda las herramientas y estrategias digitales para mejorar la evaluación. El área 5, por otro lado, gira en torno al uso de herramientas digitales para empoderar al alumnado y, finalmente, el área 6 se centra en cómo facilitar la competencia digital de los estudiantes.

Cabe reseñar que, a excepción de la primera y la última de las áreas, las demás podrían considerarse el núcleo pedagógico del marco ya que en ellas se detallan las competencias que el profesorado debe poseer para fomentar la adopción y/o adaptación de estrategias de aprendizaje efectivas, inclusivas e innovadoras, utilizando herramientas digitales.

Aunque en este estudio no profundizaremos en ellos, señalamos también la importancia de otros Marcos Europeos de Referencia como lo son el DigCompOrg y el DigComp v2.1. Ambos, al igual que el DigCompEdu, dan origen a distintas iniciativas desarrolladas y validadas por la Comisión Europea, y permiten hacer un análisis de la realidad de los centros, el alumnado y el profesorado en materia de competencias profesionales y organizacionales.

Ya en el contexto español, el Marco Común de Competencia Digital Docente (INTEF, 2017) es el marco de referencia para el diagnóstico y la mejora de las competencias digitales del profesorado. Estas competencias digitales, se definen como competencias que necesita desarrollar el profesorado del siglo XXI para la mejora de su práctica educativa y para el desarrollo profesional continuo. Describe, de manera estandariza$\mathrm{da}$, la competencia digital de los docentes en cinco áreas y tres dimensiones (básica que incluyen los niveles A1 y A2; intermedia, en la que se incluyen los niveles B1 y B2 y avanzada: niveles C1 y C2)

En la siguiente figura, se relacionan las 5 áreas propuestas en el MCCDD, como así también, las competencias incluidas en ellas.

TABLA 1. Áreas y competencias del Marco Común de Competencia Digital Docente

\begin{tabular}{ll}
\hline Áreas competenciales & Competencias \\
\hline $\begin{array}{l}\text { Área } 1 \\
\text { Información y alfabetización }\end{array}$ & $\begin{array}{l}\text { 1.1. Navegación, búsqueda y filtrado de información, datos y contenidos digitales } \\
\text { informacional }\end{array}$ \\
\hline $\begin{array}{l}\text { 1.3. Almacenamiento y recuperación de información, datos y contenidos digitales } \\
\text { Área } 2\end{array}$ & $\begin{array}{l}\text { 2.1. Interacción mediante las tecnologías digitales } \\
\text { 2.2. Compartir información y contenidos digitales }\end{array}$ \\
& $\begin{array}{l}\text { 2.3. Participación ciudadana en línea } \\
\text { 2.4. Colaboración mediante canales digitales }\end{array}$ \\
& 2.5. Netiqueta \\
& 2.6. Gestión de la identidad digital \\
\hline
\end{tabular}




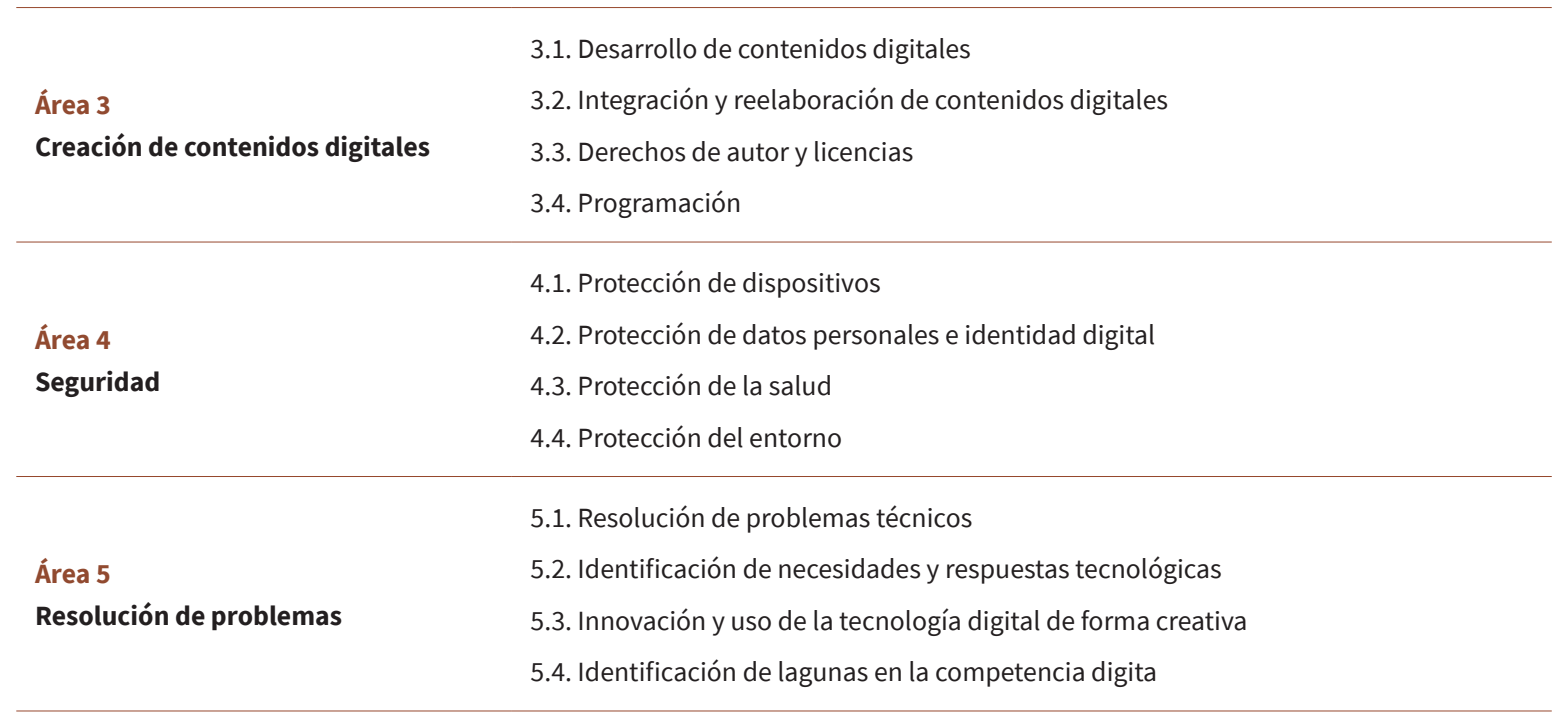

Sin lugar a duda, este Marco Común de Competencia Digital Docente (MCCD) sienta las bases para avanzar en propuestas que orienten y ayuden al profesorado, centros educativos $y$ entidades formativas $y / 0$ acreditadoras establecidas en todo el territorio español, a desarrollar instrumentos que validen están competencias.

La propuesta de una rúbrica de evaluación basada en dimensiones o áreas, indicadores y niveles de desarrollo de habilidades, hacen que este marco se convierta en un referente para el diagnóstico y la mejora de las competencias digitales del profesorado y que varias comunidades autónomas e investigadores, tomen la iniciativa para elevar propuestas capaces de concretar en procedimientos para la evaluación, desarrollo y/ o formación en materia digital. Tal es el caso, del reciente trabajo de Cabero y Palacios (2020) quienes ven compatible con el documento DigCompEdu, el Marco Común de Competencia Digital Docente propuesto por INTEF y desarrollan una herramienta de auto-reflexión para docentes denominada "DigCompEdu Check-In" y cuyos objetivos se centran en favorecer a los educadores, una mejor comprensión de este marco, a la vez que proporcionarles, una forma de autoevaluar sus fortalezas y necesidades o áreas de mejora de aprendizaje digital.

Concluir este apartado, ratificando la apuesta por la formación del personal docente en materia de competencia digital. En acuerdo con lo que señalan Durán et al. (2019), la competencia digital es clave en el desempeño de la profesión docente.

\section{LOS NIVELES DE COMPETENCIA DIGITAL DOCENTE EN LA ACTUALIDAD}

Como hemos podido observar, más allá del interés personal por desarrollar su competencia digital, los docentes de Educación Infantil y Educación Primaria cumplen con una responsabilidad fundamental en el desarrollo de las destrezas tecnológicas de los estudiantes en estas etapas, asumiendo el reto de asumir un doble papel: $\mathrm{n}$ al ser ciudadanos y formadores digitales (López-Gil, \& Bernal, 2018). Esta función capacitadora tiene, tanto un carácter instrumental, como un carácter de formación crítica, requiriendo de los profesionales unas capacidades avanzadas que les permitan acometer su función docente.

Esta cuestión supone un desafío importante porque son diversos los estudios que han puesto de manifiesto el bajo nivel de competencia digital de los docentes tanto en formación como en ejercicio en las dife- 
rentes etapas educativas. Además, existen factores como la edad que parece guardar relación con el nivel de competencia digital (Esteve-Mon et al., 2016; López et al., 2020), no pudiendo demostrarse de manera generalizada la incidencia de otros factores como el sexo del docente. En esta línea, podemos encontrar estudios que avalan el mayor nivel de competencia de las mujeres en la creación de contenidos digitales y los hombres en la resolución de problemas (Pozo et al., 2020) y en seguridad (Grande-de Prado et al., 2020).

Analizando en primer lugar la situación en los docentes en formación, los estudios avalan que los futuros maestros y maestras de Educación Infantil y Primaria cuentan con un nivel medio de competencia digital docente. Las mayores destrezas se encuentran en habilidades de gestión de la información y las competencias comunicativas y de colaboración (Torres et al., 2020), especialmente en las tareas más básicas (Moreno-Guerrero et al., 2020). Por el contrario, las mayores dificultades se asocian a la creación de contenido (Hernández, \& San Nicolás, 2019), el uso crítico, la solución de problemas, la creatividad o la innovación (Gutiérrez, \& Cabero, 2016).

Por otro lado, parece no haber consenso en relación al desarrollo de la competencia digital a medida que avanza el plan de estudios. Mientras que Gabarda et al. (2017) concluían que las destrezas de los docentes en formación mejoraban según el curso, otros estudios como el de Girón et al. (2019) no haya diferencias en este sentido. Asimismo, estos autores concluyen que no existen diferencias significativas en función de la titulación que se cursa, contando con destrezas similares los estudiantes de ambas titulaciones (Grado en Educación Infantil y Grado en Educación Primaria).

Es reseñable, para finalizar, que son diversos los estudios (Moreno et al., 2018; Pinto-Santos et al., 2020) que avalan las diferencias entre la percepción que tienen los estudiantes sobre sus niveles de competencia digital y las destrezas reales, pudiendo deberse a su manejo diario de la tecnología y a una actitud positiva frente a ella (Sánchez-Caballé et al., 2019).

En el caso del profesorado en ejercicio, la situación no mejora demasiado. En general, la literatura apunta a que el nivel de competencia digital es medio.

Las mayores fortalezas se asocian a competencias instrumentales básicas ligadas con la utilización de recursos y espacios más clásicos (Palacios-Rodríguez, \& Martín-Párraga, 2021). Estos mismos autores identifican que, sin embargo, tienen dificultades para colaborar, compartir y generar conocimiento, a las que se suman otras como la carencia de destrezas para utilizar las tecnologías para atender a la diversidad, la seguridad (Garcia-Grau et al., 2020) y la creación de contenidos digitales (Fuentes et al., 2019).

A diferencia de los docentes en formación, estudios como el de Moreno-Guerrero et al. (2020) sí evidencian algunas diferencias en función de la etapa educativa. Por un lado, todos ellos tienen un nivel alto en identificación de lagunas de la competencia digital y un nivel bajo en relación a derechos de autor y licencia. Sin embargo, en otras áreas, como compartir información y contenidos digitales o integración y reelaboración de contenidos, los docentes de Infantil cuentan con un mayor nivel.

\section{A MODO DE CONCLUSIÓN}

El análisis realizado en este trabajo ha permitido poner de relieve las demandas competenciales que los sistemas realizan a los docentes y contraponerlas con la formación inicial que reciben y con sus niveles de competencia digital reales. 
La realidad parece bastante evidente: la formación que reciben los futuros docentes de Educación Infantil y Primaria resulta a todas luces insuficiente para el abordaje de sus responsabilidades curriculares y, especialmente, para los estándares internacionales comúnmente aceptados por la comunidad académica.

Parece claro que los docentes, tanto en formación como en ejercicio son capaces de realizar tareas de carácter básico (relacionadas con cuestiones de alfabetización informacional y de comunicación), mostrando dificultades de manera manifiesta para la creación de contenidos, la seguridad o la resolución de problemas. Esta cuestión no es baladí, si retomamos una de las ideas básicas que hemos compartido anteriormente: los docentes cumplen una doble función en relación a la competencia digital, una vinculada a su propio desarrollo y otra, respecto al desarrollo de la de los estudiantes.

Resulta crucial, en este sentido, reforzar, mediante diferentes medios, las destrezas tecnológicas en los Grados de Educación Infantil y Primaria. Por un lado, mediante un compromiso institucional que establezca un nuevo marco sobre el que diseñar los planes de estudio y permitan superar las deficiencias de la normativa actual (Herrada, \& Herrada, 2011; Peirats et al., 2018). En este sentido, consideramos que sin una apuesta en firme por una mayor inclusión de contenidos y destrezas digitales en la Orden que regula el diseño de los planes de estudio de los estudios de Magisterio, se pone en riesgo el fomento de la competencia digital de los futuros docentes.

Dado que esta primera necesidad queda fuera del marco de actuación de las instituciones de Educación Superior y sus docentes, reclamamos también la necesidad de que, desde las universidades podamos seguir promoviendo el desarrollo de la competencia digital mediante acciones específicas. De este modo, se pueden establecer cauces de comunicación y colaboración mediante recursos digitales (Domingo-Coscolla et al., 2019) o invertir en acciones más complejas, como el diseño de actividades formativas mediadas por la tecnología, que contribuyan no solo al desarrollo de la competencia digital, sino al resto de destrezas básicas. En esta línea, puede considerarse la propuesta de Romero-García et al. (2020) quienes demostraron el potencial de las metodologías activas en el desarrollo de las destrezas tecnológicas. En esta misma línea, Cuevas et al. (2021) estudiaron el impacto de la gamificación en el desarrollo de competencias comunicativas, instrumentales y pedagógicas, concluyendo sus beneficios para la mejora de la competencia digital, obteniéndose resultados similares en la utilización del videoblog (Colomo et al., 2020; Vidal et al., 2020) o el flipped classroom (Colomo et al., 2020) como recursos de aprendizaje en educación superior

No queremos finalizar sin poner de manifiesto que, aunque son evidentes las carencias en la formación inicial, es realmente reseñable la acción que se realiza desde el ámbito de la formación continua para la formación tecnológica de los docentes y el desarrollo de su competencia digital, tanto a nivel nacional (a través del Instituto Nacional de Tecnologías y Formación del Profesorado) como a nivel autonómico mediante los centros de formación del profesorado de cada una de las autonomías.

En este sentido, consideramos que, únicamente desde una óptica de trabajo colaborativo y coordinado se podrá hacer frente a una necesidad tan latente y urgente como la capacitación de los docentes en su dimensión digital. 


\section{REFERENCIAS}

Aristizabal, P., \& Cruz, E. (2018). Desarrollo de la Competencia Digital en la Formación Inicial del profesorado de Educación Infantil. Pixel-Bit. Revista de Medios y Educación, (52), 97-110. http://dx.doi.org/10.12795/pixelbit.2018.i52.07

Cabero-Almenara, J., \& Palacios-Rodríguez, A. (2020). Marco Europeo de Competencia Digital Docente «DigCompEdu» y cuestionario «DigCompEdu Check-In». EDMETIC, Revista de Educa ción Mediática y TIC, 9(1), 213-234. https://doi.org/10.21071/ edmetic.v9i1.12462

Colomo, E., Gabarda, V., Cívico, A., \& Cuevas, N. (2020). Percepción de estudiantes sobre el uso del videoblog como recurso digital en educación superior. Píxel-Bit. Revista De Medios Y Educación, (59), 7-25.https://doi.org/10.12795/pixelbit.74358

Colomo, E. Soto, R., Ruiz, J., \& Gómez, M. (2020). University Students' Perception of the Usefulness of the Flipped Classroom Methodology. Education Sciences, 10(10), e275. https://doi. $\operatorname{org} / 10.3390 /$ educsci10100275

Comisión Europea (2006). Recomendación 2006/962/CE del Parlamento Europeo y del Consejo, de 18 de diciembre de 2006, sobre las competencias clave para el aprendizaje permanente. Diario Oficial de la Unión Europea, de 30 de diciembre de 2006. https://eur-lex.europa.eu/legal-content/ES/ TXT/?uri=celex\%3A32006H0962

Comisión Europea (2018). Recomendación 2018/C 189/01 del Consejo de 22 de mayo de relativa a las competencias clave para el aprendizaje permanente. Diario Oficial de la Unión Europea, de 4 de junio de 2018. https://eur-lex.europa.eu/legalcontent/ES/TXT/PDF/?uri=CELEX:32018H0604(01)\&from=SV

Cuevas, N., Cívico, A., Gabarda, V., \& Colomo, E. (2021). Percepción del alumnado sobre la gamificación en la educación superior. ReiDoCrea. Revista de investigación y Docencia Creativa, 10(16), 1-12. https://doi.org/10.30827/Digibug.66757

Cuevas, N.., Gabarda, V., Cívico, A., \& Colomo, E. (2020). Flipped classroom in COVID-19 times: a cross-talking perspective. IJERI: International Journal of Educational Research and Innovation, (15), 326-341. https://doi.org/10.46661/ijeri.5439
Domingo-Coscollola, M., Bosco-Paniagua, A., Carrasco-Segovia, S., \& Sánchez-Valero, J.A. (2019). Fomentando la competencia digital docente en la universidad: Percepción de estudiantes y docentes. Revista De Investigación Educativa, 38(1), 167182. https://doi.org/10.6018/rie.340551

Durán, M.C., Prendes, M.P., \& Guriérrez, I.P. (2019). Certificación de la Competencia Digital Docente: propuesta para el profesorado universitario. RIED. Revista Iberoamericana de Educación a Distancia, 22(1), 187-205. http://dx.doi.org/10.5944/ ried.22.1.22069

Esteve-Mon, F., Gisbert-Cervera, M,, \& Lázaro-Cantabrana, J.L (2016). La competencia digital de los futuros docentes: ¿cómo se ven los actuales estudiantes de educación? Perspectiva Educacional, Formación de Profesores, 55(2), 38-54. https://doi.org/10.4151/07189729-Vol.55-Iss.2-Art.412

Ferrari, A. (2013). DIGCOMP: A framework for developing and understanding digital competence in Europe. https://ec.europa. eu/jrc/en/publication/digcomp-framework-developing-andunderstanding-digital-competence-europe

Fuentes, A., López, J., \& Pozo, S. (2019). Análisis de la Competencia Digital Docente. Factor clave para el Desempeño de Pedagogías Activas con Realidad Aumentada. REICE: Revista Iberoamericana sobre Calidad, Eficacia y Cambio en Educación, 17(2), 27-42. https://doi.org/10.15366/reice2019.17.2.002

Gabarda, V., Marín, D., \& Romero, M.M. (2020). La competencia digital en la formación inicial docente. Percepción de los estudiantes de Magisterio de la Universidad de Valencia. ENSAYOS, Revista de la Facultad de Educación de Albacete, 35(2), 1-16. https://doi.org/10.18239/ensayos.v35i2.2176

Gabarda, V., Rodríguez, A., \& Moreno, M. D. (2017). La competencia digital en estudiantes de magisterio. Análisis competencial y percepción personal del futuro maestro. Educatio Siglo XXI, 35(2), 253-274. https://doi.org/10.6018/j/298601

Garcia i Grau, F.; Lázaro, J.L., \& Valls, C. (2020). Avanzando hacia la madurez digital del centro educativo: un análisis de la competencia digital docente. En E. Sánchez, E. Colomo, J. Ruiz 
y J. Sánchez (Coords.). Tecnologías Educativas y Estrategias Didácticas (pp. 188-199). UMA Editorial.

Girón, V., Cózar, R., \& González-Calero, J.A. (2019). Análisis de la autopercepción sobre el nivel de competencia digital docente en la formación inicial de maestros/as. Revista Electrónica Interuniversitaria De Formación Del Profesorado, 22(3), 193218. https://doi.org/10.6018/reifop.373421

Grande-de-Prado, M., Cañón-Rodríguez, R., \& García-Martín, S. (2020). Digital security, how do teachers in training perceived themselves?. IJERI: International Journal of Educational Research and Innovation, (14), 262-275. https://doi. org/10.46661/ijeri.3983

Gutiérrez, J.J., \& Cabero, J. (2016). Estudio de caso sobre la autopercepción de la competencia digital del estudiante universitario de las titulaciones de grado de Educación Infantil y Primaria. Profesorado. Revista de currículum y formación del profesorado, 20(2), 180-199.

Hernández, V. M., \& San Nicolás, M.B. (2019). Percepción del alumnado universitario sobre su grado de competencia digital. Hamut 'ay, 6(1), 7-18. http://dx.doi.org/10.21503/hamu. $\underline{\text { v6i1. } 1571}$

Herrada, R.I., \& Herrada, G. (2011). Adaptación de los estudios de magisterio al EEES. Las TIC en los nuevos planes de estudio. EDUTEC, Revista Electrónica de Tecnología Educativa, (36), $1-12$.

Instituto Nacional de Tecnologías Educativas y Formación del Profesorado - INTEF (2017). Marco Común de Competencia Digital Docente. INTEF

International Society for Tecnhnology in Education (2008) National educational technology standards for teachers (NETS). http://www.eduteka.org/pdfdir/EstandaresNETSDocentes2008.pdf

Ley 14/1970, de 4 de agosto, General de Educación y Financiamiento de la Reforma Educativa. Boletín Oficial del Estado, 187, de 6 de agosto de 1970. https://www.boe.es/buscar/doc. php?id=BOE-A-1970-852

Ley Orgánica 1/1990, de 3 de octubre, de Ordenación General del Sistema Educativo. Boletín Oficial del Estado, 238, de 4 de octubre de 1990. https://www.boe.es/eli/es/lo/1990/10/03/1
Ley Orgánica 2/2006, de 3 de mayo, de Educación. Boletín Oficial del Estado, 106, de 4 de mayo de 2006. https://www.boe.es/ eli/es/lo/2006/05/03/2

Ley Orgánica 8/2013, de 9 de diciembre, para la mejora de la calidad educativa. Boletín Oficial del Estado, 295, de 10 de diciembre de 2013. https://www.boe.es/buscar/pdf/2013/BOEA-2013-12886-consolidado.pdf

Ley Orgánica 3/2020, de 29 de diciembre, por la que se modifica la Ley Orgánica 2/2006, de 3 de mayo, de Educación. Boletín Oficial del Estado, 340, de 30 de diciembre de 2020. https:// www.boe.es/eli/es/lo/2020/12/29/3

López, J., Pozo, S., Vázquez, E., \& López, E.J. (2020). Análisis de la incidencia de la edad en la competencia digital del profesorado preuniversitario español. Revista Fuentes, 22(1), 75-87. http://doi.org/10.12795/revistafuentes.2020.v22.i1.07

López-Gil, M., \& Bernal, C. (2018). El perfil del profesorado en la Sociedad Red: reflexiones sobre la competencia digital de los y las estudiantes en Educación de la Universidad de Cádiz. IJERI: International Journal of Educational Research and Innovation, (11), 83-100.

Moreno, M.D., Gabarda, V., \& Rodríguez, A. (2018). Alfabetización informacional y competencia digital en estudiantes de magisterio. Profesorado. Revista de currículum y formación del profesorado, 22(3), 253-270. https://doi.org/10.30827/profesorado.v22i3.8001

Moreno-Guerrero, A.J., López-Belmonte, J., Pozo, S., \& López, J.A. (2020). Estado de la competencia digital docente en las distintas etapas educativas desde un alcance internacional. Revista Espacios, 41(26). http://www.revistaespacios.com/ a20v41n16/a20v41n16p19.pdf

Moreno-Guerrero, A.J., Miaja-Chippirraz, N., Bueno-Pedrero, A., \& Borrego-Otero, L. (2020). El área de información y alfabetización informacional de la competencia digital docente. Revista Electrónica Educare, 24(3), 521-536. https://dx.doi. org/10.15359/ree.24-3.25

Orden $\mathrm{ECl} / 3854 / 2007$, de 27 de diciembre, por la que se establecen los requisitos para la verificación de los títulos universitarios oficiales que habiliten para el ejercicio de la profesión de Maestro en Educación Infantil. Boletín Oficial del Estado, 
312, de 29 de diciembre de 2007. https://www.boe.es/eli/ es/o/2007/12/27/eci3854

Orden $\mathrm{ECl} / 3857 / 2007$, de 27 de diciembre por la que se establecen los requisitos para la verificación de los títulos universitarios oficiales que habiliten para el ejercicio de la profesión de Maestro en Educación Primaria. Boletín Oficial del Estado, 312, de 29 de diciembre de 2007. https://www.boe.es/eli/ es/o/2007/12/27/eci3857

Organización de las Naciones Unidas para la Educación, la Ciencia y la Cultura (2008). Normas sobre competencias en TIC para docentes. http://www.eduteka.org/pdfdir/UNESCOEstandaresDocentes.pdf

Organización de las Naciones Unidas para la Educación, la Ciencia y la Cultura (2019). Marco de competencias de los docentes en materia de TIC. https://unesdoc.unesco.org/ark:/48223/ pf0000371024

Palacios-Rodríguez, A., \& Martín-Párraga, L. (2021). Formación del profesorado en la era digital. Nivel de innovación y uso de las TIC según el Marco Común de referencia de la Competencia digital docente. Revista De Investigación Y Evaluación Educativa, 8(1), 38-53. https://doi.org/10.47554/revie2021.8.79

Peirats, J., Marín, D., Granados, J., \& Morote, D. (2018). Competencia digital en los planes de estudio de universidades públicas españolas. REDU. Revista de Docencia Universitaria, 16(1), 175-191. https://doi.org/10.4995/redu.2018.8935

Pinto-Santos, A.R., Pérez, A., \& Darder, A. (2020). Autopercepción de la competencia digital docente en la formación inicial del profesorado de educación infantil. Revista Espacios, 41(18). https://www.revistaespacios.com/a20v41n18/ a20v41n18p29.pdf

Pozo, S., López, J., Fernández, M., \& López, J. A. (2020). Análisis correlacional de los factores incidentes en el nivel de competencia digital del profesorado. Revista Electrónica Interuniversitaria De Formación Del Profesorado, 23(1), 143-159. https://doi.org/10.6018/reifop.396741

Ramírez, A., Morales, A.T., \& Olguín, P.A. (2015). Marcos de referencia de saberes digitales. EDMETIC; Revista de Educación Mediática y TIC, 4(2), 112- 136
Real Decreto 126/2014, de 28 de febrero, por el que se establece el currículo básico de la Educación Primaria. Boletín Oficial del Estado, 52, de 1 de marzo de 2014. http://www.boe.es/ buscar/doc.php?id=BOE-A-2014-2222

Real Decreto 1513/2006, de 7 de diciembre, por el que se establecen las enseñanzas mínimas de la Educación primaria. Boletín Oficial del Estado, 293, de 9 de diciembre de 2006. https:// www.boe.es/eli/es/rd/2006/12/07/1513/con

Real Decreto 1630/2006, de 29 de diciembre, por el que se establecen las enseñanzas mínimas del segundo ciclo de Educación infantil. Boletín Oficial del Estado, 4, de 4 de enero de 2007. https://www.boe.es/eli/es/rd/2006/12/29/1630

Redecker, C., \& Punie, Y. (2017). Digital Competence of Educators DigCompEdu. Publications Office of the European Union

Romero-García, C., Buzón-García, O., Sacristán, M., \& Navarro, E. (2020). Evaluación de un programa para la mejora del aprendizaje y la competencia digital en futuros docentes empleando metodologías activas. Estudios Sobre Educación, 39, 179205. http://doi.org/10.15581/004.39.179-205

Sánchez-Caballé, A., Gisbert-Cervera, M., \& Esteve-Mon, F. (2019). La competencia digital de los estudiantes universitarios de primer curso de grado. Innoeduca. International Journal of Technology and Educational Innovation, 5(2), 104-113. https://doi.org/10.24310/innoeduca.2019.v5i2.5598

Torres, A., Jiménez, D., González, V., Martínez, M.A., \& Morales, J. (2020). La competencia digital de los futuros docentes de secundaria y su mejora a partir de la formación específica en TIC. En R. Roig-Vila (ed.). La docencia en la Enseñanza Superior. Nuevas aportaciones desde la investigación e innovación educativas (pp. 445-453). Octaedro.

Vidal, M.I., Marín, D., \& Pardo, M.I. (2020). El edublog en la formación inicial de docentes: espacio para la construcción de conocimientos compartidos. ReiDoCrea. Revista de investigación y Docencia Creativa, 9, 212-219. http://doi. org/10.30827/Digibug.66362 